\title{
Estratégias fisioterapêuticas para tratamento de pacientes portadores de doença de
} Alzheimer: Revisão de literatura

\author{
Physiotherapeutic strategies for the treatment of patients with Alzheimer's disease: Literature \\ review
}

Estrategias fiosterapéuticas para el tratamiento de pacientes com enfermedad de Alzheimer: Revisión de la literatura

Recebido: 12/07/2021 | Revisado: 21/07/2021 | Aceito: 30/07/2021 | Publicado: 05/08/2021

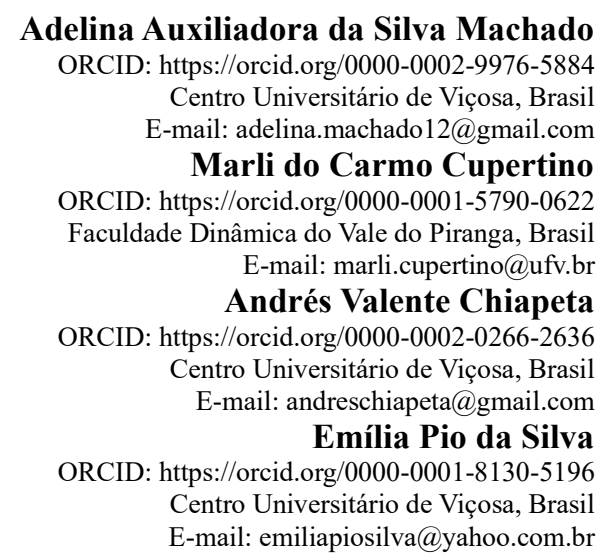

\begin{abstract}
Resumo
$\mathrm{O}$ aumento da expectativa da população brasileira tem permitido que as pessoas alcancem uma longevidade maior. Este fato não implica necessariamente em uma velhice saudável, já que nessa fase da vida torna-se bastante comum a presença das doenças crônicas não transmissíveis, dentre elas a Doença de Alzheimer. A fisioterapia é uma ferramenta importante na melhora da qualidade de vida e no reestabelecimento funcional e motor dos portadores de Alzheimer, com o objetivo de retardar o avanço da doença, preservando a funcionalidade motora o mais próximo do normal, atuando com uma equipe multidisciplinar, orientando aos familiares e cuidadores do portador da demência. O objetivo do presente estudo foi especificar as intervenções fisioterapêuticas mais utilizadas durante a reabilitação de pacientes portadores de Doença de Alzheimer e descrever como as intervenções fisioterapêuticas contribuem para a melhoria e manutenção do desempenho funcional dos pacientes. Trata-se de uma pesquisa bibliográfica descritiva e de caráter qualitativo. As buscas foram realizadas pelas bases de dados Biblioteca Virtual de Saúde (BVS), PEDro e PUBMED e os descritores utilizados foram: Alzheimer, Fisioterapia, Dementia de Alzheimer, Rehabilitation, Physioteraphy. Foram selecionados 7 artigos científicos para análise e discussão dos resultados que obedeceram aos critérios de inclusão e exclusão da pesquisa. A partir dos resultados obtidos, conclui-se que a terapia baseada em exercícios físicos é eficaz nos tratamentos não farmacológicos para pacientes diagnosticados com Doença de Alzheimer.
\end{abstract}

Palavras-chave: Doença de Alzheimer; Intervenções fisioterapêuticas; Fisioterapia; Reabilitação.

\begin{abstract}
The increase in life expectancy of the Brazilian population has allowed people to achieve greater longevity. This fact does not necessarily imply a healthy old age, since at this stage of life the presence of non-communicable chronic diseases, including Alzheimer's disease, is quite common. Physiotherapy is an important tool in improving the quality of life and in the functional and motor reestablishment of Alzheimer's patients, with the objective of delaying the progress of the disease, preserving motor functionality as close to normal as possible, working with a multidisciplinary team, guiding family members and caregivers of dementia patients. The aim of the present study was to specify the most used physical therapy interventions during the rehabilitation of patients with Alzheimer's Disease and to describe how physical therapy interventions contribute to the improvement and maintenance of the patients' functional performance. This is a descriptive and qualitative bibliographic research. Searches were performed using the Virtual Health Library (BVS), PEDro and PUBMED databases and the descriptors used were: Alzheimer, Physiotherapy, Alzheimer's Dementia, Rehabilitation, Physioteraphy. Seven scientific articles were selected for analysis and discussion of the results that met the inclusion and exclusion criteria for the research. From the results obtained, it can
\end{abstract}


be concluded that exercise-based therapy is effective in non-pharmacological treatments for patients diagnosed with Alzheimer's Disease.

Keywords: Alzheimer's disease; Physiotherapeutic interventions; Physiotherapy; Rehabilitation.

\section{Resumen}

El aumento de la esperanza de vida de la población brasileña ha permitido que las personas alcancen una mayor longevidad. Este hecho no implica necesariamente una vejez saludable, ya que en esta etapa de la vida es bastante común la presencia de enfermedades crónicas no transmisibles, incluida la enfermedad de Alzheimer. La fisioterapia es una herramienta importante en la mejora de la calidad de vida y en el restablecimiento funcional y motor de los pacientes con Alzheimer, con el objetivo de retrasar el avance de la enfermedad, preservando la funcionalidad motora lo más cercana a la normalidad, trabajando con un equipo multidisciplinar, orientando familiares y cuidadores de pacientes con demencia. El objetivo del presente estudio fue especificar las intervenciones de fisioterapia más utilizadas durante la rehabilitación de pacientes con enfermedad de Alzheimer y describir cómo las intervenciones de fisioterapia contribuyen a la mejora y mantenimiento del rendimiento funcional de los pacientes. Se trata de una investigación bibliográfica descriptiva y cualitativa. Las búsquedas se realizaron en la Biblioteca Virtual en Salud (BVS), las bases de datos PEDro y PUBMED y los descriptores utilizados fueron: Alzheimer, Fisioterapia, Alzheimer Demencia, Rehabilitación, Fisioterapia. Se seleccionaron siete artículos científicos para el análisis y discusión de los resultados que cumplieron con los criterios de inclusión y exclusión de la investigación. De los resultados obtenidos se puede concluir que la terapia basada en ejercicio es eficaz en tratamientos no farmacológicos para pacientes diagnosticados de Alzheimer.

Palabras clave: Enfermedad de Alzheimer; Intervenciones fisioterapéuticas; Fisioterapia; Rehabilitación.

\section{Introdução}

O aumento da expectativa de vida da população brasileira tem permitido que as pessoas alcancem uma longevidade maior, pois é fato que o brasileiro tem vivido mais, o que necessariamente não implica em uma velhice saudável, já que nessa fase da vida torna-se bastante comum a presença das doenças crônicas não transmissíveis, dentre elas destaca-se a Doença de Alzheimer (Bitencourt et al., 2018). Estimativas da Organização Mundial da Saúde (OMS, 2010) revelaram que 18 milhões de homens e mulheres sofrem de Alzheimer, e a projeção é de que esse número possa quase que dobrar em 2025, atingindo cerca de 34 milhões de pessoas (De Aquino et al., 2013). No Brasil estima-se que 1,2 milhões de pessoas sofrem com esta enfermidade (OMS, 2019) e que os custos com estes pacientes podem comprometer dois terços da renda de uma família (Veras, et al., 2008).

A Doença de Alzheimer (DA) é definida como uma doença neurodegenerativa que se manifesta apresentado deterioração cognitiva e da memória recente, manifestando também uma variedade de sintomas neuropsiquiátricos e de alterações comportamentais que se agravam ao decorrer do tempo (ABRAZ, 2018). É a causa mais comum de demência, correspondendo por 50 a 75\% dos casos (Alzheimer's Disease International, 2015).

Segundo a Associação Brasileira de Alzheimer (ABRAZ, 2018), a causa da patologia ainda é desconhecida, porém, estudos indicam que ela seja geneticamente determinada. A doença tem início com uma replicação anormal de determinadas proteínas do sistema nervoso central (SNC), gerando proteínas geneticamente alteradas que levam toxicidade aos neurônios e aos espaços que há entre eles; a consequência dessa toxicidade é a perda progressiva de neurônios em determinadas regiões do encéfalo.

O tratamento da DA tem como objetivo adiar ao máximo a evolução e as complicações decorrentes da doença; seja de forma farmacológica, através de medicamentos; ou não farmacológica, por meio da fisioterapia, a fim de reestabelecer funções cognitivas e motoras perdidas, assim como melhorar a qualidade de vida $(\mathrm{QV})$ dos portadores de Doença de Alzheimer (Marinho, 2020).

No tratamento do paciente com DA, medidas não farmacológicas são adotadas; como a estimulação de pacientes apáticos, visando a melhor estruturação do tempo, da ocupação e do meio ambiente do paciente; elaboração de programas para o cotidiano e o desenvolvimento de rotinas que contribuem para seu bem-estar (Leite et al., 2014). 
Conhecendo os comprometimentos causados na DA, a fisioterapia tem o papel de auxiliar no tratamento do paciente. A reabilitação ajuda a retardar o processo da doença, evitar encurtamentos musculares e deformidades articulares. Além disso, incentiva a independência do paciente, oferece orientações familiares para o cuidado (Medeiros et al., 2016).

A fisioterapia é uma ferramenta importante na melhora da qualidade devida e no reestabelecimento funcional e motor dos portadores de DA, apesar de não existir um tratamento preventivo e nem curativo para a doença. Sabe-se ainda que o paciente necessita de uma abordagem multidisciplinar para a sua reabilitação global (Carvalho et.al., 2008).

De acordo com Bitencourt (2018), na fisioterapia, independente da modalidade utilizada para terapêutica, é imprescindível que o paciente seja avaliado e que seja elaborado um plano de tratamento adequado e individualizado para os portadores da doença de Alzheimer, de acordo com a funcionalidade e estágio da doença.

Segundo com Lima et al. (2016), o tratamento fisioterapêutico consiste em um programa de exercícios de resistência e fortalecimento que são importantes para aumentar e manter a força muscular assim como melhorar o metabolismo. Os exercícios de alongamento são capazes de auxiliar na manutenção ou no ganho de flexibilidade. A reabilitação envolve ainda atividades relacionadas ao equilíbrio, treino de marcha e à prevenção de quedas.

A intervenção fisioterapêutica atuará conforme a necessidade de cada paciente; com o objetivo de retardar o avanço da doença, preservando a funcionalidade motora o mais próximo do normal, atuando com uma equipe multidisciplinar, orientando aos familiares e cuidadores do portador da demência (Zaions et al., 2012).

Desse modo, Colombo et al. (2009) afirma que a intervenção fisioterapêutica pode contribuir em qualquer fase da doença, ao atuar tanto na manutenção quanto na melhora do desempenho funcional do indivíduo, para mantê-lo mais ativo e independente possível, sendo importante ressaltar que esses fatores contribuem diretamente na qualidade de vida do paciente portador de Doença de Alzheimer.

Diante do exposto, o presente estudo teve como objetivo especificar as estratégias fisioterapêuticas mais utilizadas durante a reabilitação de pacientes portadores de Doença de Alzheimer e descrever como essas estratégias contribuem para a melhoria e a manutenção do desempenho funcional dos pacientes.

\section{Metodologia}

Trata-se de uma pesquisa bibliográfica descritiva e de caráter qualitativo. Para Brevidelli e Sertório (2010) uma pesquisa descritiva é a aquela onde os resultados são expressos em narrativas, figuras, quadros, descrições e que a abordagem qualitativa busca detectar os significados que as pessoas dão aos fenômenos. Para realização da pesquisa foram adotadas as seguintes etapas: I) busca de artigos em banco de dados científicos para aprofundamento do tema; II) leitura exploratória dos artigos identificados; III) aplicação dos critérios de inclusão ou exclusão; IV) análise, interpretação e construção dos resultados.

A busca na literatura foi realizada através das bases de dados científicos Biblioteca Virtual de Saúde (BVS), PEDro e PUBMED e os descritores utilizados foram: Alzheimer, Fisioterapia, Dementia de Alzheimer, Rehabilitation, Physiotherapy.

O recorte temporal foi de estudos publicados nos últimos 10 anos e que tinham relação com o objetivo da pesquisa. Os critérios de inclusão adotados foram artigos publicados nos idiomas português e inglês, em periódicos nacionais e internacionais, disponíveis gratuitamente na íntegra. Ao mesmo tempo, foram excluídos, durante a busca, artigos que não estão relacionados com intervenções fisioterapêuticas na doença de Alzheimer e que foram publicados há mais de 10 anos, além dos publicados em outros idiomas e artigos de revisão de literatura.

Os dados obtidos a partir dos artigos selecionados foram inseridos em uma tabela criada exclusivamente para a pesquisa, utilizando o programa Microsoft Office Excel® 2013. Tais dados foram analisados de forma crítica e apresentados em forma de resultados em quadro e fluxograma. 


\section{Resultados e Discussão}

Foram encontrados 40 artigos, sendo 18 da BVS e 22 da PUBMED. Destes, cinco (5) estavam duplicados nas referidas bases de dados, restando 35 artigos na literatura, dos quais 28 foram excluídos por não se adequarem aos critérios de exclusão e inclusão. Deste modo, a revisão bibliográfica foi construída com 7 artigos científicos (Figura 1). Já o Quadro 1 mostra a distribuição dos artigos inclusos na revisão bibliográfica.

Figura 1. Fluxograma dos estudos identificados e selecionados para construção da revisão bibliográfica.

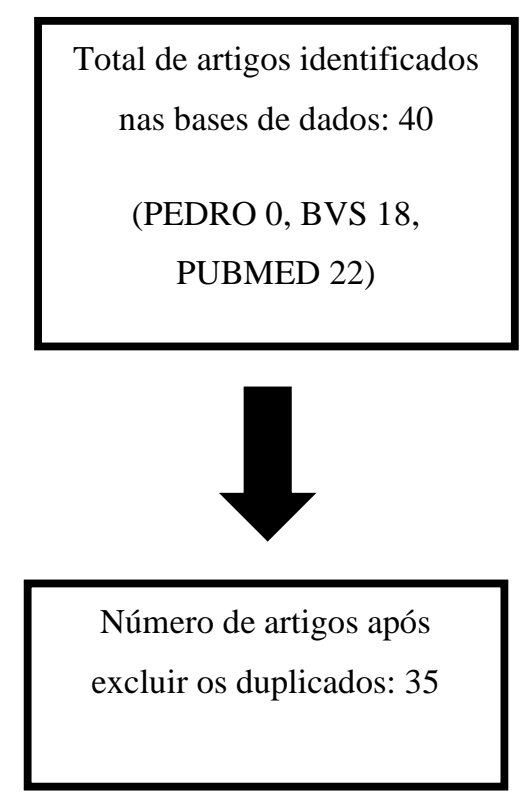

Fonte: Autoria Própria (2021)
Número de artigos excluídos: 28, onde:

13 = não abordavam o tema.

4 = não disponíveis na integra.

$6=$ artigos de revisão

$2=$ idiomas diferentes

$3=$ artigos de estudo de protocolos

Número de artigos em textos completos avaliados para elegibilidade: 7

Quadro 1. Descrição dos artigos elegíveis.

\begin{tabular}{|c|c|c|c|c|c|}
\hline Título/Autor/Ano & $\begin{array}{l}\text { Objetivo do } \\
\text { Estudo }\end{array}$ & $\begin{array}{c}\text { Metodologia } \\
\text { Utilizada }\end{array}$ & $\begin{array}{c}\text { Intervenção } \\
\text { Utilizada }\end{array}$ & Resultados & Conclusão \\
\hline $\begin{array}{l}\text { The effect of } \\
\text { stimulation } \\
\text { therapy and donepezil } \\
\text { on cognitive function in } \\
\text { Alzheimer's disease. A } \\
\text { community based RCT } \\
\text { with a two-by-two } \\
\text { factorial design. } \\
\text { Andersen et al. (2012) }\end{array}$ & $\begin{array}{l}\text { Examinar o efeito } \\
\text { da terapia de } \\
\text { estimulação na } \\
\text { função cognitiva } \\
\text { em moradores da } \\
\text { comunidade com } \\
\text { DA leve a } \\
\text { moderada no norte } \\
\text { da Noruega. } \\
\text { Buscou-se } \\
\text { examinar se o } \\
\text { Donepezil } \\
\text { aumentava o efeito } \\
\text { da terapia de } \\
\text { estimulação na } \\
\text { cognição. }\end{array}$ & $\begin{array}{l}\text { Realizado um estudo } \\
\text { fatorial em nove } \\
\text { municípios rurais no } \\
\text { norte da Noruega, } \\
\text { dois por dois } \\
\text { compreendendo } \\
\text { terapia de } \\
\text { estimulação por um } \\
\text { ano em comparação } \\
\text { com o padrão } \\
\text { cuidado ao qual um } \\
\text { ensaio randomizado } \\
\text { duplo-cego } \\
\text { controlado por } \\
\text { placebo com } \\
\text { donepezil foi } \\
\text { adicionado. A } \\
\text { amostra foi } \\
\text { composta por } 187 \\
\text { participantes com } 65 \\
\text { anos ou mais com } \\
\text { um diagnóstico } \\
\text { recente de DA leve } \\
\text { ou moderada, dos } \\
\text { quais } 146\end{array}$ & $\begin{array}{l}\text { Terapia de } \\
\text { estimulação incluindo } \\
\text { atividades físicas, } \\
\text { estimulação } \\
\text { cognitiva, sensorial e } \\
\text { social. O programa } \\
\text { incluiu } \\
\text { atividades } \\
\text { cotidianas realizadas } \\
\text { de forma } \\
\text { sistemática } \\
\text { intensificada como } \\
\text { caminhada, trabalho } \\
\text { doméstico, } \\
\text { leitura regular de } \\
\text { livros e jornais, } \\
\text { treinamento em sala } \\
\text { de ginástica, } \\
\text { dança, palavras } \\
\text { cruzadas, ouvir } \\
\text { música e participação } \\
\text { regular na } \\
\text { vida social da } \\
\text { comunidade. } \\
\text { Algumas atividades }\end{array}$ & $\begin{array}{l}\text { As pontuações do } \\
\text { Mini-Mental-State } \\
\text {-Examination } \\
\text { (MMSE) } \\
\text { permaneceram } \\
\text { inalteradas entre os } \\
\text { participantes com } \\
\text { DA que receberam } \\
\text { terapia de } \\
\text { estimulação e } \\
\text { aqueles } \\
\text { recebendo cuidados } \\
\text { padrão. }\end{array}$ & $\begin{array}{l}\text { Portadores de DA } \\
\text { recentemente } \\
\text { diagnosticada } \\
\text { recebendo } \\
\text { terapia de } \\
\text { estimulação reteve } \\
\text { a função cognitiva } \\
\text { durante } \\
\text { o acompanhamento } \\
\text { de um ano, assim } \\
\text { como os } \\
\text { participantes DA } \\
\text { que receberam } \\
\text { cuidado padrão e } \\
\text { que a terapia com } \\
\text { donepezila não teve } \\
\text { efeito na cognição. }\end{array}$ \\
\hline
\end{tabular}




\begin{tabular}{|c|c|c|c|c|c|}
\hline & & $\begin{array}{l}\text { completaram um } \\
\text { acompanhamento de } \\
\text { um ano. }\end{array}$ & $\begin{array}{l}\text { mais sofisticadas } \\
\text { como grupos de } \\
\text { reminiscências, } \\
\text { Sudoku, } \\
\text { aromaterapia } \\
\text { e jardim sensorial } \\
\text { foram } \\
\text { adicionadas, entre as } \\
\text { quais os } \\
\text { participantes } \\
\text { podiam se mover } \\
\text { livremente. }\end{array}$ & & \\
\hline $\begin{array}{l}\text { Treadmill training as } \\
\text { an } \\
\text { augmentation } \\
\text { treatment } \\
\text { for Alzheimer's } \\
\text { disease: } \\
\text { a pilot randomized } \\
\text { controlled study. } \\
\text { Arcoverde } \\
\text { al .(2013) }\end{array}$ & $\begin{array}{l}\text { Avaliar o efeito do } \\
\text { exercício aeróbio } \\
\text { na cognição e na } \\
\text { capacidade } \\
\text { funcional em } \\
\text { pacientes com } \\
\text { Doença de } \\
\text { Alzheimer (DA). }\end{array}$ & $\begin{array}{l}\text { Foi realizado um } \\
\text { estudo por quatro } \\
\text { meses, controlado, } \\
\text { randomizado e } \\
\text { simples-cedo. Foram } \\
\text { selecionados } 20 \\
\text { idosos com } \\
\text { demência leves, } \\
\text { separados em dois } \\
\text { grupos: Grupo } \\
\text { exercício (GE) } \\
\text { realizavam esteira } \\
30 \text { minutos ,2 vezes } \\
\text { na semana e com } \\
\text { intensidade } \\
\text { moderada) e grupo } \\
\text { controle (GC). }\end{array}$ & $\begin{array}{l}\text { A intervenção foi } \\
\text { dividida em três } \\
\text { fases: 1) Exercícios de } \\
\text { aquecimento na } \\
\text { esteira por dez } \\
\text { minutos } \\
\text { intensidade de 40\% } \\
\text { do VO2máx; 2) } 20 \\
\text { minutos a uma } \\
\text { intensidade de } 60 \% \\
\text { VO2máx; } 3 \text { ) } 5 \\
\text { minutos de exercícios } \\
\text { de } \\
\text { alongamento } \\
\text { supervisionado } \\
\text { focados nos grandes } \\
\text { músculos } \\
\text { grupos. Além disso, } \\
\text { eram dados } \\
\text { comandos } \\
\text { como como verbais } \\
\text { olhar para frente, para } \\
\text { se segurar } \\
\text { a esteira, dar passos } \\
\text { largos, não } \\
\text { falar, manter postura } \\
\text { adequada. }\end{array}$ & $\begin{array}{l}\text { Após } 16 \text { semanas, } \\
\text { o GE mostrou } \\
\text { melhora na } \\
\text { cognição enquanto } \\
\text { o GC declinou. } \\
\text { Comparado ao GC, o } \\
\text { GE apresentou } \\
\text { melhora significativa } \\
\text { na capacidade } \\
\text { funcional. }\end{array}$ & $\begin{array}{l}\text { Caminhar na esteira } \\
\text { pode ser } \\
\text { recomendado como } \\
\text { um tratamento } \\
\text { adicional para } \\
\text { pacientes com doença } \\
\text { de } \\
\text { Alzheimer. }\end{array}$ \\
\hline $\begin{array}{l}\text { Comparative } \\
\text { Cognitive } \\
\text { Effects } \\
\text { of Choreographed } \\
\text { Exercise and } \\
\text { Multimodal Physical } \\
\text { Therapy in Older } \\
\text { Adults with Amnestic } \\
\text { Mild } \\
\text { Cognitive } \\
\text { Impairment: } \\
\text { Randomized } \\
\text { Clinical Trial } \\
\text { Bisbe et al. (2019) }\end{array}$ & $\begin{array}{l}\text { Comparar os } \\
\text { efeitos cognitivos } \\
\text { do exercício } \\
\text { coreografado com } \\
\text { um exercício físico } \\
\text { multimodal num } \\
\text { programa de } \\
\text { terapia em idosos } \\
\text { com } \\
\text { comprometimento } \\
\text { cognitivo leve } \\
\text { (CCL). }\end{array}$ & $\begin{array}{l}\text { Foi realizado um } \\
\text { ensaio clinico } \\
\text { randomizado com } \\
\text { dois grupos } \\
\text { paralelos sob sigilo } \\
\text { de alocação e } \\
\text { avaliador cego. Os } \\
\text { participantes foram } \\
\text { divididos em grupo } \\
\text { Coreografia ou } \\
\text { Fisioterapia e } \\
\text { realizaram os } \\
\text { exercícios duas } \\
\text { vezes por semana } \\
\text { em sessões de } 60 \\
\text { minutos durante } 12 \\
\text { semanas. }\end{array}$ & $\begin{array}{l}\text { No grupo } \\
\text { Coreografia:12 vídeos } \\
\text { com tutoriais e com } \\
\text { variedades de } \\
\text { estilos musicais } \\
\text { (salsa, rock, } \\
\text { zumba, pop, etc). } \\
\text { Grupo } \\
\text { Fisioterapia: } \\
\text { exercícios físicos } \\
\text { seguindo } \\
\text { multimodal } \\
\text { programa } \\
\text { fisioterapia com } \\
\text { diferentes habilidades } \\
\text { motoras } \\
\text { como } \\
\text { resistência, } \\
\text { flexibilidade, } \\
\text { equilíbrio, } \\
\text { coordenação } \\
\text { marcha. }\end{array}$ & $\begin{array}{l}\text { O estudo mostrou } \\
\text { que houve } \\
\text { diferenças } \\
\text { significativas nos } \\
\text { resultados físicos e } \\
\text { cognitivos. Ambos } \\
\text { grupos melhoram } \\
\text { significativamente } \\
\text { em termos visuais } \\
\text { recordação } \\
\text { atrasada. O grupo } \\
\text { Coreografia exibiu } \\
\text { significamente mais } \\
\text { benefícios na } \\
\text { memória e } \\
\text { reconhecimento } \\
\text { verbal e do que o } \\
\text { grupo Fisioterapia. }\end{array}$ & $\begin{array}{l}\text { O estudo fornece } \\
\text { evidencias sobre } \\
\text { com diferentes } \\
\text { formas de exercício } \\
\text { melhoram as } \\
\text { funções } \\
\text { neuropsicológicas e } \\
\text { funções físicas. } \\
\text { Porém, maiores } \\
\text { benefícios } \\
\text { cognitivos foram } \\
\text { alcançados na } \\
\text { intervenção } \\
\text { coreográfica do que } \\
\text { na intervenção } \\
\text { fisioterapêutica } \\
\text { multimodal. }\end{array}$ \\
\hline $\begin{array}{l}\text { Structured physical } \\
\text { exercise improves } \\
\text { neuropsychiatric } \\
\text { symptoms in acute } \\
\text { dementia care: a } \\
\text { hospital-based RCT. } \\
\text { Fleiner et al. (2017) }\end{array}$ & $\begin{array}{l}\text { Investigar os } \\
\text { efeitos de um } \\
\text { programa de } \\
\text { exercícios de curto } \\
\text { prazo sobre } \\
\text { sinais e sintomas } \\
\text { neuropsiquiátricos } \\
\text { no tratamento da } \\
\text { demência hospitalar } \\
\text { aguda. }\end{array}$ & $\begin{array}{l}\text { Foi realizado um } \\
\text { ensaio clínico } \\
\text { randomizado } \\
\text { controlado com base } \\
\text { em um hospital. Os } \\
\text { pacientes foram } \\
\text { divididos de forma } \\
\text { aleatória em GC } \\
\text { (grupo controle) e GI } \\
\text { (grupo de } \\
\text { intervenção), o grupo }\end{array}$ & $\begin{array}{l}\text { O cronograma de } \\
\text { exercícios } \\
\text { constituía exercícios } \\
\text { de } \\
\text { fortalecimento com } \\
\text { pesos no tornozelo ou } \\
\text { no pulso ou } \\
\text { exercícios } \\
\text { resistência para } \\
\text { membros inferiores e } \\
\text { superiores }\end{array}$ & $\begin{array}{l}\text { Em comparação } \\
\text { com o GC, o GI } \\
\text { apresentou redução } \\
\text { significativa nos } \\
\text { sinais e sintomas } \\
\text { neuropsiquiátricos. } \\
\text { Especialmente, o } \\
\text { comportamento } \\
\text { agitado e a labilidade } \\
\text { melhoraram. Não } \\
\text { houve }\end{array}$ & $\begin{array}{l}\text { O programa de } \\
\text { exercícios é } \\
\text { facilmente aplicável } \\
\text { em } \\
\text { tratamento de } \\
\text { demência hospitalar } \\
\text { e reduz } \\
\text { significativamente a } \\
\text { neuropsiquiatria } \\
\text { sinais e sintomas } \\
\text { em pacientes que }\end{array}$ \\
\hline
\end{tabular}




\begin{tabular}{|c|c|c|c|c|c|}
\hline & & $\begin{array}{l}\text { de intervenção } \\
\text { conduziu um } \\
\text { exercício de } 2 \\
\text { semanas } \\
\text { programa com } \\
\text { quatro sessões de } \\
\text { exercícios de } 20 \\
\text { minutos em } 3 \text { dias } \\
\text { por semana. O grupo } \\
\text { de controle conduziu } \\
\text { uma estimulação } \\
\text { social } \\
\text { programa. Os efeitos } \\
\text { sobre os sinais e } \\
\text { sintomas } \\
\text { neuropsiquiátricos } \\
\text { foram medidos } \\
\text { através da } \\
\text { Cooperativa de } \\
\text { Doença de } \\
\text { Alzheimer } \\
\text { Estudo-Clinical } \\
\text { Global Impression of } \\
\text { Change, o } \\
\text { Neuropsychiatric } \\
\text { Inventory e a Cohen- } \\
\text { Mansfield Agitation } \\
\text { Inventário. }\end{array}$ & $\begin{array}{l}\text { em ergômetros } \\
\text { sentados no GI. } \\
\text { Já no GC foi realizado } \\
\text { um } \\
\text { programa de } \\
\text { estimulação social de } \\
\text { mesa assistida de } \\
\text { jogos, que } \\
\text { foram instruídos por } \\
\text { terapeutas } \\
\text { ocupacional do } \\
\text { hospital. }\end{array}$ & $\begin{array}{l}\text { diferenças entre os } \\
\text { grupos em relação } \\
\text { a medicamentos } \\
\text { antipsicóticos e } \\
\text { benzodiazepínicos }\end{array}$ & $\begin{array}{l}\text { sofrem de } \\
\text { estágios } \\
\text { predominantemente } \\
\text { moderados de } \\
\text { demência. }\end{array}$ \\
\hline $\begin{array}{l}\text { A controlled clinical } \\
\text { trail on the } \\
\text { Effects of exercise on } \\
\text { neuropsychiatric } \\
\text { disorders and } \\
\text { instrumental } \\
\text { activities in } \\
\text { women with } \\
\text { Alzheimer's } \\
\text { Disease. } \\
\text { Nascimento et al. } \\
(2012)\end{array}$ & $\begin{array}{l}\text { Analisar os efeitos } \\
\text { de um programa } \\
\text { de exercícios } \\
\text { multimodal } \\
\text { associado à } \\
\text { estimulação } \\
\text { cognitiva nos } \\
\text { distúrbios } \\
\text { neuropsiquiátricos } \\
\text { e no desempenho } \\
\text { de atividades } \\
\text { funcionais em } \\
\text { idosas com } \\
\text { Doença de } \\
\text { Alzheimer (DA). }\end{array}$ & $\begin{array}{l}27 \text { mulheres } \\
\text { portadoras de DA } \\
\text { recrutadas por meio } \\
\text { de anúncios em } \\
\text { jornais, televisão e } \\
\text { rádio. Divididas em } \\
\text { dois grupos de } \\
\text { acordo com sua } \\
\text { vontade de seguir no } \\
\text { programa de } \\
\text { exercícios: grupo } \\
\text { controle (12) e grupo } \\
\text { experimental (15). } \\
\text { Os participantes } \\
\text { eram assistidos por } \\
\text { um programa } \\
\text { interdisciplinar que } \\
\text { consistia em terapia } \\
\text { de estimulação } \\
\text { cognitiva, terapia } \\
\text { ocupacional e } \\
\text { educação física. As } \\
\text { sessões eram } \\
\text { realizadas três vezes } \\
\text { por semana durante } \\
\text { uma hora, incluindo } \\
\text { atividade aeróbica e } \\
\text { diferentes tipos de } \\
\text { atividades que } \\
\text { beneficiariam a } \\
\text { capacidade } \\
\text { funcional. }\end{array}$ & 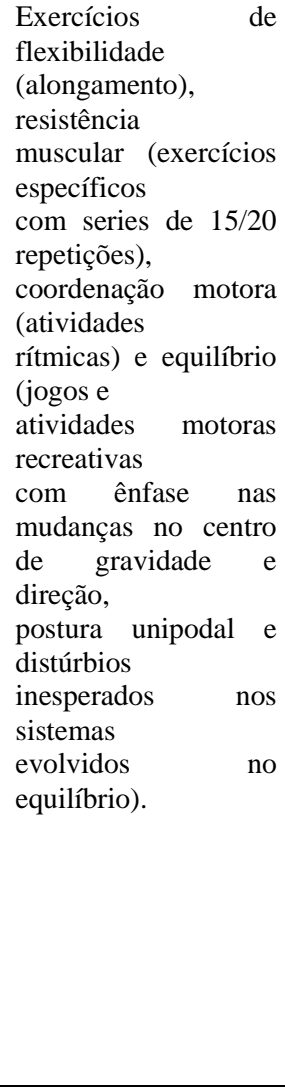 & $\begin{array}{l}\text { Para as mulheres } \\
\text { com DA que } \\
\text { realizaram seis } \\
\text { meses do programa } \\
\text { de exercício } \\
\text { multimodal, houve } \\
\text { redução significativa } \\
\text { nos distúrbios } \\
\text { neuropsiquiátricos, } \\
\text { enquanto, nas } \\
\text { mulheres do grupo } \\
\text { controle os } \\
\text { distúrbios } \\
\text { aumentam } \\
\text { ligeiramente. }\end{array}$ & $\begin{array}{l}\text { Em seis meses de } \\
\text { um programa de } \\
\text { exercícios } \\
\text { multimodal, houve } \\
\text { redução dos } \\
\text { sintomas } \\
\text { neuropsiquiátricos } \\
\text { em pacientes com DA } \\
\text { e contribuiu para } \\
\text { a atenuação do } \\
\text { comprometimento } \\
\text { no desempenho das } \\
\text { atividades } \\
\text { instrumentais da } \\
\text { vida diária em } \\
\text { idosas com DA. }\end{array}$ \\
\hline $\begin{array}{l}\text { Effects of a } \\
\text { multidisciplinar } \\
\text { cognitive } \\
\text { rehabilitation } \\
\text { program for } \\
\text { patients with mild } \\
\text { Alzheimer's disease. } \\
\text { Viola et al. (2011) }\end{array}$ & $\begin{array}{l}\text { Avaliar os efeitos } \\
\text { de um programa } \\
\text { de reabilitação } \\
\text { multidisciplinar na } \\
\text { cognição, } \\
\text { qualidade de vida } \\
\text { e } \\
\text { sintomas } \\
\text { neuropsiquiátricos } \\
\text { em pacientes com } \\
\text { doença de } \\
\text { Alzheimer leve. }\end{array}$ & $\begin{array}{l}\text { Foram selecionados } \\
25 \text { pacientes com } \\
\text { DA e AVC e a } \\
\text { doença de Alzheimer } \\
\text { e seus respectivos } \\
\text { cuidadores foram } \\
\text { recrutados. Foi } \\
\text { realizado um estudo } \\
\text { simples-cego, } \\
\text { controlado e } \\
\text { conduzido num }\end{array}$ & $\begin{array}{l}\text { Reabilitação } \\
\text { cognitiva por meio de } \\
\text { exercícios para } \\
\text { melhorar a } \\
\text { atenção, memória, } \\
\text { espaço e } \\
\text { orientação temporal e } \\
\text { auto- } \\
\text { adaptações cognitivas } \\
\text { imparidade. } \\
\text { treinamento }\end{array}$ & $\begin{array}{l}\text { O tratamento foi } \\
\text { benéfico para a } \\
\text { qualidade de vida } \\
\text { dos pacientes e } \\
\text { cuidadores. As } \\
\text { intervenções } \\
\text { utilizadas foram } \\
\text { associadas a } \\
\text { redução dos } \\
\text { sintomas de } \\
\text { depressão para }\end{array}$ & $\begin{array}{l}\text { O programa de } \\
\text { reabilitação } \\
\text { multimodal foi } \\
\text { benéfico para os } \\
\text { pacientes } \\
\text { portadores de DA } \\
\text { uma vez que foi } \\
\text { associado a } \\
\text { melhorias na } \\
\text { qualidade de vida } \\
\text { desses pacientes e }\end{array}$ \\
\hline
\end{tabular}




\begin{tabular}{|c|c|c|c|c|c|}
\hline & & $\begin{array}{l}\text { hospital-dia de uma } \\
\text { universidade. O } \\
\text { estudo durou um } \\
\text { período de } 12 \\
\text { semanas de } \\
\text { estimulação e } \\
\text { programa } \\
\text { psicoeducacional. }\end{array}$ & $\begin{array}{l}\text { cognitivo assistido } \\
\text { por } \\
\text { computador consistia } \\
\text { principalmente em } \\
\text { jogos de } \\
\text { memória e atenção. } \\
\text { Terapia de } \\
\text { arte que visa estimular } \\
\text { a } \\
\text { cognição, habilidades } \\
\text { emocionais } \\
\text { interpessoais por } \\
\text { meio de } \\
\text { habilidades } \\
\text { expressivas e } \\
\text { técnicas artísticas, } \\
\text { terapia } \\
\text { ocupacional que tinha } \\
\text { como } \\
\text { objetivo desenvolver } \\
\text { recursos e } \\
\text { estratégias } \\
\text { melhorar a } \\
\text { conclusão para } \\
\text { funcional. E } \\
\text { fisioterapia que teve } \\
\text { como } \\
\text { objetivo melhorar o } \\
\text { equilíbrio e } \\
\text { prevenção de quedas } \\
\text { por meio de } \\
\text { exercícios } \\
\text { administrados aos } \\
\text { pacientes. } \\
\text { treinamento físico No } \\
\text { foi oferecido um } \\
\text { programa } \\
\text { complementar para } \\
\text { pacientes } \\
\text { fisicamente capazes } \\
\text { de melhorar } \\
\text { o condicionamento } \\
\text { físico. Ênfase } \\
\text { especial foi colocada } \\
\text { em aspectos } \\
\text { motores, emocionais, } \\
\text { sociais e } \\
\text { cognitivos. } \\
\text { Além de exercícios de } \\
\text { força e } \\
\text { equilíbrio, } \\
\text { pacientes foram } \\
\text { convidados } \\
\text { caminhadas em para } \\
\text { grupo e sessões de } \\
\text { alongamento. } \\
\end{array}$ & $\begin{array}{l}\text { paciente e para os } \\
\text { cuidadores e houve } \\
\text { diminuição nos } \\
\text { sintomas } \\
\text { neuropsiquiátricos } \\
\text { em pacientes } \\
\text { portadores de DA. }\end{array}$ & $\begin{array}{l}\text { que também foi } \\
\text { observado uma } \\
\text { diminuição } \\
\text { significativa na } \\
\text { depressão e } \\
\text { sintomas de } \\
\text { sobrecarga no } \\
\text { cuidador. }\end{array}$ \\
\hline $\begin{array}{l}\text { Cognitive change is } \\
\text { more } \\
\text { positively associated } \\
\text { with } \\
\text { an active lifestyle } \\
\text { than } \\
\text { with training } \\
\text { interventions } \\
\text { in older adults at risk } \\
\text { of } \\
\text { dementia: } \\
\text { controlled } \\
\text { interventional clinical } \\
\text { trial. } \\
\text { Küster et al. (2016) }\end{array}$ & $\begin{array}{l}\text { Avaliar } \\
\text { e comparar as } \\
\text { mudanças } \\
\text { cognitivas } \\
\text { relacionadas ao } \\
\text { treinamento e ao } \\
\text { estilo de vida em } \\
\text { idosos com } \\
\text { demência. }\end{array}$ & $\begin{array}{l}54 \text { idosos em risco } \\
\text { de demência foram } \\
\text { recrutados para } 10 \\
\text { semanas de } \\
\text { treinamento físico, } \\
\text { cognitivo ou } \\
\text { colocados em } \\
\text { condição de controle } \\
\text { em uma lista de } \\
\text { espera. O estilo de } \\
\text { vida foi avaliado com } \\
\text { vários testes antes e } \\
\text { após as } \\
\text { intervenções, bem } \\
\text { como } \\
\text { acompanhamento }\end{array}$ & $\begin{array}{l}\text { O treinamento } \\
\text { cognitivo consistia } \\
\text { em sessões de } 1 \text { hora } \\
\text { cinco } \\
\text { vezes } \\
\text { por semana durante } \\
10 \text { semanas. } \\
\text { O treinamento } \\
\text { consistia em seis } \\
\text { tarefas diferentes que } \\
\text { visam a } \\
\text { discriminação } \\
\text { auditiva de } \\
\text { frequências e sílabas, } \\
\text { bem como }\end{array}$ & $\begin{array}{l}\text { Nenhuma das } \\
\text { intervenções de } \\
\text { treinamento } \\
\text { melhorou a } \\
\text { cognição global, em } \\
\text { contrapartida, o } \\
\text { estilo de vida } \\
\text { autorrelatado foi } \\
\text { positivamente } \\
\text { associado a } \\
\text { benefícios na } \\
\text { cognição. Além } \\
\text { disso, a associação } \\
\text { de estilo de vida } \\
\text { ativo com mudança } \\
\text { cognitiva foi }\end{array}$ & $\begin{array}{l}\text { As associações de } \\
\text { um estilo de vida } \\
\text { ativo com mudança } \\
\text { cognitiva ao longo } \\
\text { do tempo em um } \\
\text { grupo de risco de } \\
\text { demência foram } \\
\text { mais forte do que os } \\
\text { efeitos de } \\
\text { intervenções de } \\
\text { treinamento } \\
\text { específico de curto } \\
\text { prazo. Estes } \\
\text { resultados } \\
\text { demonstram que um } \\
\text { estilo de vida ativo }\end{array}$ \\
\hline
\end{tabular}




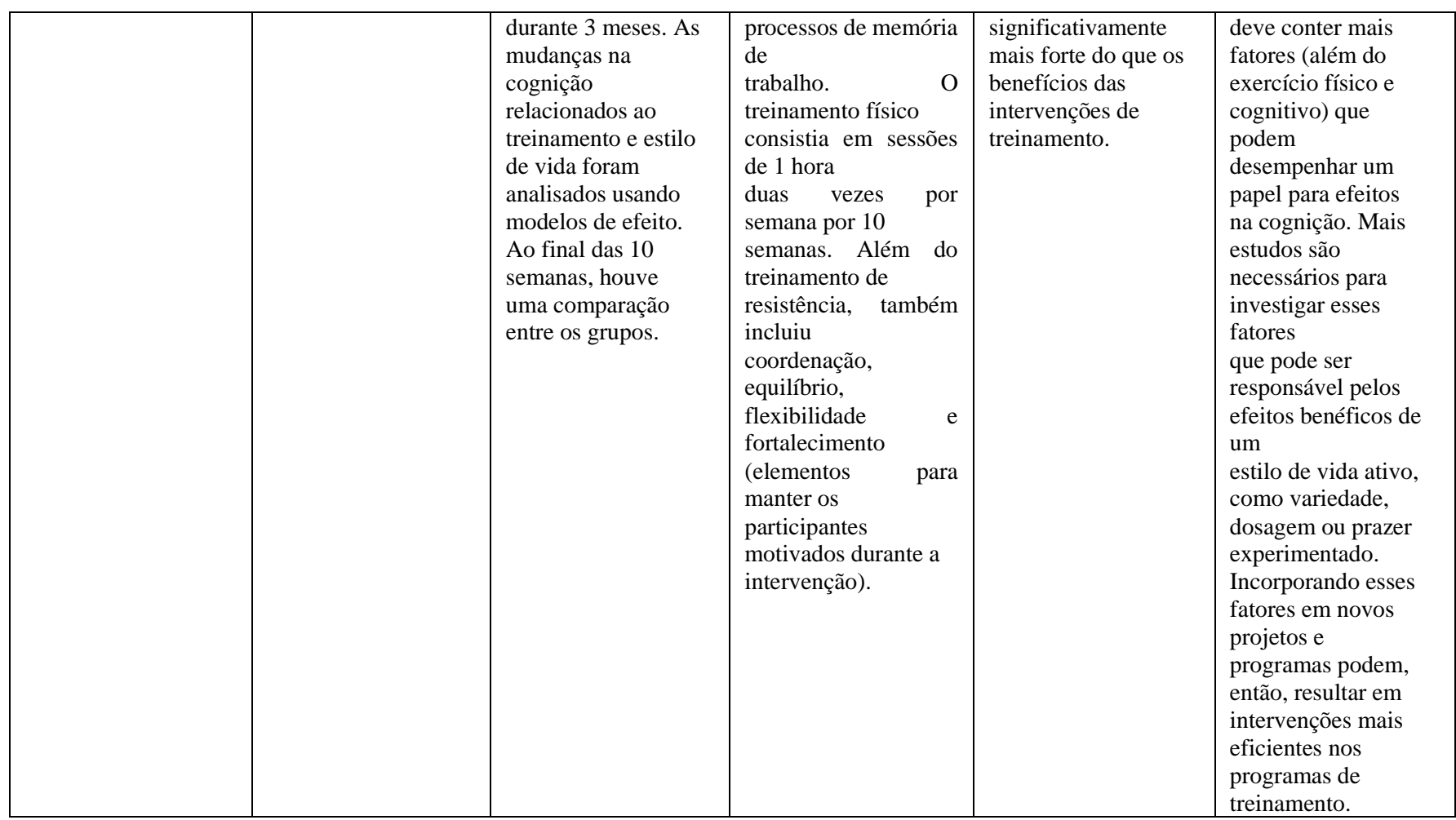

Fonte: Autoria Própria (2021).

No estudo realizado por Nascimento et al. (2012), os participantes eram atendidos por um programa interdisciplinar em que consistia terapia de estimulação cognitiva, terapia ocupacional e educação física. A intervenção utilizada abordava exercícios de dupla tarefa flexibilidade (alongamento), resistência muscular (exercícios específicos com séries de 15/20 repetições), coordenação motora (atividades rítmicas) e equilíbrio (jogos e atividades motoras recreativas) com ênfase nas mudanças no centro de gravidade e direção, postura unipodal e distúrbios inesperados nos sistemas envolvidos no equilíbrio. Os resultados obtidos demonstraram que mulheres com DA que realizaram exercícios multimodal tiveram redução significativa nos distúrbios neuropsiquiátricos em relação às mulheres que não foram submetidas a esse tipo de intervenção.

Dados semelhantes foram encontrados no estudo de Arcoverde et al. (2013). O autor evidenciou por meio de seu estudo que caminhar na esteira pode ser recomendado como um tratamento adicional para pacientes com DA. Para chegar nessa conclusão, o autor avaliou o efeito do exercício aeróbico (caminhar na esteira) na cognição e na capacidade funcional de 20 pacientes idosos com DA, que foram divididos em dois grupos. Um grupo de exercícios que realizavam esteira por 30 minutos, duas vezes na semana e com intensidade moderada e o grupo controle que não realizava esse tipo de exercício. Além disso, eram dados comandos verbais como olhar para frente para se segurar na esteira, dar passos largos, não falar e manter a postura adequada. Deste modo, em 16 semanas o grupo de exercícios mostrou melhora na cognição, enquanto os participantes do grupo controle declinaram. E, em comparação entre o grupo controle e grupo de exercícios, o de controle apresentou melhora significativa na capacidade funcional, principalmente, se tratando de equilíbrio e risco de queda. Os estudos anteriores mostraram que houve melhora no desempenho funcional como um todo.

Diferentemente do que foi encontrado nos estudos de Arcoverde et al. (2013) e Nascimento et al. (2012), o estudo de Andersern et al. (2012) abordou terapia de estimulação incluindo atividades físicas, estimulação cognitiva, sensorial e social. Ainda foram inclusas nesse programa de tratamento, atividades cotidianas realizadas de forma sistemática e intensificada; como caminhada, trabalho doméstico, leitura regular de livros e jornais, treinamento em sala de ginástica, dança, palavras cruzadas, ouvir música e participação regular na vida social da comunidade e algumas atividades mais sofisticadas (Sudoku, aromaterapia, jardim sensorial, entre outras). Através do teste Mini Mental State (MMSE), pode-se observar que as pontuações permaneceram 
intactas entre os participantes que receberam a terapia de estimulação e aqueles que receberam cuidados padrão, portanto, portadores de DA recebendo a terapia, preservou a função cognitiva durante o acompanhamento de um ano, assim como os participantes com DA que receberam somente os cuidados padrão.

Entretanto, já no estudo de Viola et al. (2011), não foi encontrado melhora na capacidade cognitiva dos pacientes com DA que faziam parte do grupo de intervenção do programa de reabilitação multidisciplinar; que consistia em exercícios para melhorar a atenção, memória, espaço e orientação temporal e auto adaptações cognitivas. O treinamento cognitivo foi assistido por um computador e compreendia-se principalmente por jogos de memória e atenção, terapia de arte que visava estimular a cognição, habilidades emocionais e interpessoais por meio de habilidades expressivas e técnicas artísticas, terapia ocupacional e que tinham como objetivo, desenvolver recursos e estratégias para melhorar a conclusão funcional. A fisioterapia teve como objetivo melhorar o equilíbrio e o risco de quedas por meio de exercícios administrados aos pacientes e no treinamento físico, foi oferecido um programa complementar para pacientes fisicamente capazes de melhorar o condicionamento físico, com ênfase especial em colocar aspectos motores, emocionais, sociais e cognitivos. Além de exercícios de força e equilíbrio, os pacientes também foram convidados a realizar caminhadas em grupo e sessões de alongamento. Verificou-se que não houve declínio na capacidade funcional, assim como, aconteceu com o grupo controle, o que mostra uma influência positiva na abordagem utilizada nas funções cognitivas e no desempenho das tarefas que exigiam atenção em pacientes com DA.

O estudo de Bisbe et al. (2019) comparou os efeitos cognitivos do exercício coreografado com um exercício físico multimodal em um programa de terapia em idosos com comprometimento cognitivo leve. Por se tratar de um ensaio clínico randomizado, os participantes foram alocados em dois grupos. Grupo Fisioterapia - onde foi realizado exercícios físicos seguindo um programa de fisioterapia com habilidades motoras como força, resistência, flexibilidade, equilíbrio, coordenação e marcha; e Grupo Coreografia - onde foi gravado 12 vídeos com tutoriais e com variedades de estilos musicais (zumba, salsa, rock, pop e etc.). O estudo mostrou que houve diferenças significativas nos resultados físicos e cognitivos e que ambos grupos melhoraram de forma significativa em termos visuais de recordação atrasada. Entretanto, o grupo coreografia exibiu significativamente mais benefícios na memória e reconhecimento verbal em relação ao grupo fisioterapia. O estudo mostrou evidências sobre como diferentes formas de exercício melhoram as funções neuropsicológicas e físicas. Verificou-se que houve maiores benefícios cognitivos com a intervenção coreográfica do que na intervenção fisioterapêutica modal, isso pode-se dar ao fato de que as sessões do grupo de fisioterapia se limitavam a somente práticas de exercício físicos.

Diferentemente de todos os estudos encontrados, Fleiner et al. (2017) desenvolveram um estudo que tinha como objetivo investigar os efeitos de um programa de exercícios a curto prazo sobre sinais e sintomas neuropsiquiátricos no tratamento da demência hospitalar aguda, para tanto dividiu os participantes em dois grupos. Para o grupo de intervenção (GI) estabeleceu um programa que consistia em exercícios de fortalecimento com pesos nos tornozelos e nos pulsos ou exercícios de resistência para membros inferiores e superiores em ergômetros sentados. Já no grupo controle (GC), foi realizado um programa de estimulação social que tinha como intervenção uma mesa assistida de jogos; além disso, os pacientes foram instruídos por terapeutas ocupacionais no hospital. O estudo mostrou que em comparação com o GC, o GI apresentou uma redução significativa nos sinais e sintomas neuropsiquiátricos, em especial, no comportamento agitado e na labilidade, evidenciando que o programa de exercícios é facilmente aplicável no tratamento da demência hospitalar e que reduz significativamente sinais e sintomas em pacientes que sofrem de estágios moderados de demência.

Küster et al. (2016) realizou um estudo que buscou avaliar e comparar as mudanças cognitivas relacionadas ao treinamento e ao estilo de vida em idosos com demência. $O$ treinamento cognitivo consistiu em sessões de 1 hora, cinco vezes por semana e com duração de 10 semanas. Estava incluso no treinamento, seis tarefas diferentes que visavam a discriminação auditiva de frequências e sílabas, bem como processos de memória de trabalho. No treinamento físico, as sessões duravam 1 hora e eram realizadas duas vezes por semana em um período de 10 semanas. Também foi realizado treinamento de resistência, 
que consistia em exercícios de coordenação, equilíbrio, flexibilidade e fortalecimento. Entretanto, ao contrário dos outros estudos, nenhuma das intervenções de treinamento melhorou a cognição global dos participantes. Em contrapartida, o estilo de vida, autorrelatado, foi positivamente associado a benefícios na cognição. Além disso, a associação de estilo de vida ativo com a mudança cognitiva, foi significativamente mais forte do que os benefícios das intervenções de treinamento cognitivo, físico e de resistência. Constatou-se que o treinamento físico não impactou na melhora das funções cognitivas dos pacientes.

\section{Conclusão}

Baseado nos dados obtidos, conclui-se que a terapia baseada em exercícios físicos é eficaz nos tratamentos não farmacológicos para pacientes diagnosticados com doença de Alzheimer. Entretanto, de acordo com a literatura pesquisada não foi possível definir o tipo de exercício utilizado, a intensidade do exercício e período necessário para alcançar benefícios na capacidade funcional, no desempenho funcional e na capacidade cognitiva do paciente portador de doença de Alzheimer. O que se pode afirmar é que a fisioterapia é capaz de evitar ou diminuir complicações e deformidades, melhorar o equilíbrio, prevenir danos motores, melhorar a força muscular, treinar a realização das atividades de vida diária, assim, tentando prolongar a independência do portador de DA e melhorar sua qualidade de vida, reduzindo também sinais e sintomas da demência e melhorando as funções cognitivas.

Portanto, consideram-se necessários mais estudos, conduzidos com uma amostra maior e que utilizem técnicas específicas da fisioterapia baseada em exercício físico, estabelecendo um protocolo de atendimento com recomendações para investigar quais intervenções contribuem para a manutenção e conservação do estado cognitivo dos pacientes portadores de doença de Alzheimer.

\section{Referências}

Alzheimer's Disease International. World Alzheimer Report 2015: the global impact of dementia: an analysis of prevalence, incidence, cost and trends. London: Alzheimer's Disease International; 2015.

Andersen, F., Viitanen, M., Halvorsen, D. S., Straume, B., Wilsgaard, T., \& Engstad, T. A. (2012). The effect of stimulation therapy and donepezil on cognitive function in Alzheimer's disease. A community based RCT with a two-by-two factorial design. BMC Neurol., 12 (59), 1-10.

Arcoverde, C., Deslandes, A., Moraes, H., Almeida, C., Araujo, N. B., Vasques, P. E., Silveira, H., \& Laks, J. (2013). Treadmill training as an augmentation treatment for Alzheimer's disease: a pilot randomized controlled study. Arq Neuropsquiatr., 72 (3), 190-196.

Associação Brasileira de Alzheimer (ABRAZ). Setembro: Mês Mundial da Doença de Alzheimer. https://abraz.org.br/2020/2018/09/03/setembro-mes-mundialda-doenca-de-alzheimer/\&gt

Bisbe, M, Fuente-Vidal, A., López, E., Moreno, M., Naya, M., Benetti, C., Milá, R., Bruna, O., Boada, M., \& Alegret, M. (2020). Comparative Cognitive Effects of Choreographed Exercise and Multimodal Physical Therapy in Older Adults with Amnestic Mild Cognitive Impairment: Randomized. Clinical Trial. $J$ Alzheimers Dis, 73 (2), 769-783.

Bitencourt, E. M., Kuerten, C. M. X., Budny, J., \& Tuon, T. (2018). Doença de Alzheimer: aspectos fisiopatológicos qualidade de vida, estratégias terapêuticas da fisioterapia e biomedicina. Revista Inova Saúde, 8 (2).

Brevidelli, M. M. (2010). TCC-Trabalho de conclusão de curso: guia prático para docentes e alunos da área da saúde. Látria.

Carvalho, K. R., Cabral, R. M. C., Gomes, D. A. G. S., \& Tavares, A. B. (2008). O método Kabat no tratamento fisioterapêutico da doença de Alzheimer. Revista Kairós, 11 (2), 181-195.

Colombo Ely, J., \& Grave, M. (2008). Estratégias de intervenção fisioterapêutica em indivíduo portador de Doença de Alzheimer. Revista Brasileira de Ciências do Envelhecimento Humano, 5 (2).

De Aquino, R. G. F. (2013). Abordagem Fisioterapêutica no Paciente Portador de Doença de Alzheimer: Revisão da Literatura. CORPVS/Rev. dos Cursos de Saúde da Faculdade Integrada do Ceará, 25 (1), 39-44.

Fleiner, T., Dauth, H., Gersie, M., Zijlstra, W., \& Haussermann, P. (2017). Structured physical exercise improves neuropsychiatric symptoms in acute dementia care: a hospital-based RCT. BMC Psychiatry, 9 (68).

Küster, O. C., Fissler, P., Laptinskava, D., Thurm, F., Scharpf, A., Woll, A., Kolassa, S., Kramer, A. F., Elbert, T., Arnim, C. A. F., \& Kolassa, I. T. (2016). Cognitive change is more positively associated with an active lifestyle than with training interventions in older adults at risk of dementia: a controlled interventional clinical trial. BMC Psychiatry, 16 (1). 
Research, Society and Development, v. 10, n. 10, e83101018139, 2021

(CC BY 4.0) | ISSN 2525-3409 | DOI: http://dx.doi.org/10.33448/rsd-v10i10.18139

Leite, C. D. S. M., Menezes, T. L. M., Lyra, E. V.V., Araújo, C. M. T., et al. (2014). Conhecimento e intervenção do cuidador na doença de Alzheimer: uma revisão da literatura. J. Bras. Psiquiatr., 63 (1), 48-56.

Lima, A. M. A., Sousa, L. B., Souza, M. T. W., \& Siqueira, T. D. A. (2016). O papel da fisioterapia no tratamento da Doença de Alzheimer: uma revisão de literatura. Boletim Informativo Unimotrisaude em Sociogerontologia, 7 (1), 39.

Marinho, M. F. S. (2020). A importância da fisioterapia na Doença de Alzheimer. Environmental Smoke, 3 (1), 69-78.

Medeiros, I. M. P. J., Securella, F. F., Santos, R. C. C. S., \& Silva, K. M. R. (2015). A influência da fisioterapia na cognição de idosos com doença de Alzheimer. Revista UNILUS Ensino e Pesquisa, 12 (29).

Nascimento, C. M. C., Teixeira, C. V. L., Gobbi, L. T. B., Gobbi, S., \& Stella, F. (2012). A controlled clincal trail on the Effects of exercise on neuropsychiatric desorders and instrumental activities in women with Alzheimer's Disease. Rev. Bras. Fisioter, 16 (3).

Viola, L. F., Nunes, P. V., Yassuda, M. S., Aprahamian, I., Santos, F. S., Santos, G. D., Brum, P. S., Borges, S. M., Oliveira, A. M., Chaves, G. F. S., Ciasca, E. C., Ferreira, R. C. R., de Paula, V. J. R., Takeda, O. H., Mirandez, R. M., Watari, R., Falcao, D. V. S., Cachioni, M., \& Forlenza, O. V. (2012). Effects of a multidisciplinary cognitive rehabilitation program for patients with mild Alzheimer's disease. Clinics, 66 (8).

Veras, R. P., Caldas, C. P., Dantas, S. B., Sancho, L. G., Sicsu, B., \& Motta, L. B. (2008). Demented elderly people living at home in Rio de Janeiro, Brazil: Evaluation of expenditure on care. Psychogeriatrics, 8(2), 88-95.

World Health Organization. (2019). Risk reduction of cognitive decline and dementia: WHO guidelines.

Zaions J. D. C., Pavan, F. J., \& Wisniewski, M. S. W. (2012). A influência da fisioterapia na preservação da memória e capacidade funcional de idosos portadores da Demência de Alzheimer. Rev PERSPECTIVA, 36 (133), 151-162. 